\title{
Erratum: \\ Billiards in a General Domain with Random Reflections
}

\author{
Francis Comets, Serguei Popov, Gunter M. Schütz \\ \& MARINA VACHKOVSKAIA
}

\section{Erratum to: Arch. Rational Mech. Anal. (2009) 191:497-537 DOI 10.1007/s00205-008-0120-x}

Theorem 2.8 is incorrect as stated in the general case. The proof works only in the case $d=2$ and $\operatorname{Conv} \mathcal{D}^{\prime} \subset \mathcal{D}$, since otherwise one cannot guarantee that any chord of $\mathcal{D}$ that intersects Conv $\mathcal{D}^{\prime}$ has to intersect $\mathcal{D}^{\prime}$ as well. This makes wrong the statement between dashes on line 12 of page 535 in the proof. Below, we give the corrected versions of Theorem 2.8, the paragraph immediately after it, and its proof.

Theorem 2.8. Let $\mathcal{D}^{\prime} \subset \mathcal{D}$ be with the Lipschitz condition and an almost everywhere continuously differentiable boundary. For every Borel subset $C$ of $\partial \mathcal{D}^{\prime} \times \partial \mathcal{D}^{\prime}$, we have

$$
\mathbf{E}\left[\sum_{k=1}^{\iota} \mathbf{1}\left\{\left(\Xi_{1, k}^{\prime}, \Xi_{2, k}^{\prime}\right) \in C\right\}\right]=\frac{\left|\partial \mathcal{D}^{\prime}\right|}{|\partial \mathcal{D}|} \times\left|\partial \mathcal{D}^{\prime}\right|^{-1} \int_{C} \tilde{K}(x, y) \mathrm{d} x \mathrm{~d} y .
$$

In particular,

$$
\mathbf{E}[\iota]=\frac{\left|\partial \mathcal{D}^{\prime}\right|}{|\partial \mathcal{D}|} .
$$

Hence, the relation (16) has the following interpretation: the expected value of the measure giving unit weight to each chord induced on $\mathcal{D}^{\prime}$ by the $\mathcal{D}$-random chord, is the product of the expected number of induced chords and the probability distribution for the (ordered) endpoints of the random chord of $\mathcal{D}^{\prime}$. Another comment on this theorem is that the number $\iota$ of induced chords is integrable, a property which does not seem easy to prove directly. The formula (17) could remind the reader of the following fact concerning the so-called Poisson line process (see e.g. Section 8.4.2 of [27]): if $\mathcal{D}$ is compact and convex, then the number of lines hitting $\mathcal{D}$ has Poisson distribution with the mean proportional to the perimeter of $\mathcal{D}$.

The online version of the original article can be found under doi:10.1007/s00205-008-120-x. 
Proof of Theorem 2.8. Let $y \in \partial \mathcal{D}^{\prime}$ where $\partial \mathcal{D}^{\prime}$ has a locally $\mathcal{C}^{1}$ parametrization, $\delta>0$ and a Borel set $B \subset \mathbb{S}_{\mathbf{n}(y)}$. Similarly to (46), we get

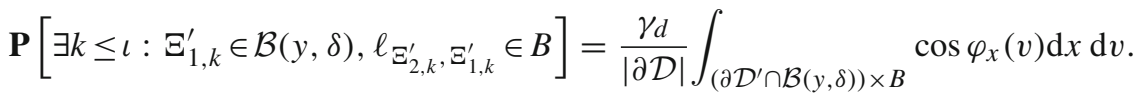

Note that, for small $\delta$, the left-hand side can be written as

$$
\mathbf{E}\left[\sum_{k=1}^{\iota} \mathbf{1}\left\{\Xi_{1, k}^{\prime} \in \mathcal{B}(y, \delta), \ell_{\Xi_{2, k}^{\prime}, \Xi_{1, k}^{\prime}} \in B\right\}\right] .
$$

Then, for $A_{1}, A_{2} \in \partial \mathcal{D}^{\prime}$ such that $A_{1} \cap A_{2}=\emptyset$ and $x \leftrightarrow y$ in $\mathcal{D}^{\prime}$ for all $x \in A_{1}$, $y \in A_{2}$, we get

$$
\mathbf{E}\left[\sum_{k=1}^{\iota} \mathbf{1}\left\{\Xi_{1, k}^{\prime} \in A_{1}, \Xi_{2, k}^{\prime} \in A_{2}\right\}\right]=\frac{1}{|\partial \mathcal{D}|} \int_{A_{1} \times A_{2}} \tilde{K}(x, y) \mathrm{d} x \mathrm{~d} y .
$$

This is enough to ensure that, for $\mathcal{C} \subset \partial \mathcal{D}^{\prime} \times \partial \mathcal{D}^{\prime}$, we have

$$
\mathbf{E}\left[\sum_{k=1}^{\iota} \mathbf{1}\left\{\left(\Xi_{1, k}^{\prime}, \Xi_{2, k}^{\prime}\right) \in C\right\}\right]=\frac{1}{|\partial \mathcal{D}|} \int_{C} \tilde{K}(x, y) \mathrm{d} x \mathrm{~d} y .
$$

which proves (16). Taking $C=\partial \mathcal{D}^{\prime} \times \partial \mathcal{D}^{\prime}$ in (16), we obtain (17).

UFR de Mathématiques, Université Paris 7, case 7012, 2, place Jussieu, 75251 Paris Cedex 05, France.

e-mail: comets@math.jussieu.fr

URL: http://www.proba.jussieu.fr/ comets

$$
\text { and }
$$

Instituto de Matemática e Estatística,

Universidade de São Paulo, rua do Matão 1010,

São Paulo, SP CEP 05508-090, Brazil.

e-mail:popov@ime.usp.br

URL: http://www.ime.usp.br/ popov

$$
\text { and }
$$

Institut für Festkörperforschung,

Forschungszentrum Jülich GmbH, 52425 Jülich, Deutschland. e-mail: G.Schuetz@fz-juelich.de

URL: http://www.fz-juelich.de/iff/staff/Schuetz_G/

and

Instituto de Matemática, Estatística e Computação Científica,

Universidade de Campinas,

Caixa Postal 6065, Campinas, SP CEP 13083-970, Brazil.

e-mail:marinav@ime.unicamp.br

URL: http://www.ime.unicamp.br/ marinav 